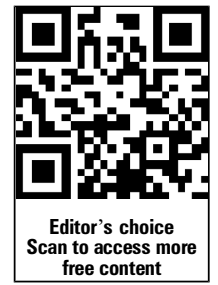

${ }^{1}$ Department of Social \& Environmental Health Research, London School of Hygiene \& Tropical Medicine, London, UK ${ }^{2}$ Department of Science, Technology, Engineering and Public Policy (STEaPP), University College London, London, UK

${ }^{3} \mathrm{NIHR}$ Collaboration for Leadership in Applied Health Research and Care in Greater Manchester, Manchester Business School, University of Manchester, Manchester, UK ${ }^{4}$ School of Social Sciences, University of Manchester, Manchester, UK

\section{Correspondence to}

Dr Theo Lorenc, Department of Science, Technology, Engineering and Public Policy (STEaPP), University College London, Gower Street, London WC1E 6BT, UK ; t.lorenc@ucl.ac.uk

Public health interventions may have a range of adverse effects, which are rarely measured or well understood.

Received 16 July 2013 Revised 12 November 2013 Accepted 21 November 2013 Published Online First 13 December 2013

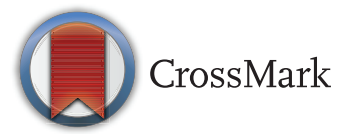

To cite: Lorenc T, Oliver $\mathrm{K}$. $J$ Epidemiol Community Health 2014;68:288-290.

\title{
Adverse effects of public health interventions: a conceptual framework
}

\author{
Theo Lorenc, ${ }^{1,2}$ Kathryn Oliver ${ }^{3,4}$
}

\section{ABSTRACT}

Public health interventions may have a range of adverse effects. However, there is limited guidance as to how evaluations should address the possibility of adverse effects. This discussion paper briefly presents a framework for thinking about the potential harms of public health interventions, focusing on the following categories: direct harms; psychological harms; equity harms; group and social harms; and opportunity harms. We conclude that the possibility of adverse effects needs to be taken into account by those implementing and evaluating interventions, and requires a broad perspective on the potential impacts of public health strategies.

\section{INTRODUCTION}

Many public health interventions may have unintended effects. The possibility of positive 'knock-on' effects beyond those envisaged by intervention developers-for example, improved social interaction as a result of environmental changes to increase walkability-is frequently discussed. However, unintended adverse effects may also frequently result from well-intentioned interventions, ${ }^{1}$ but are rarely addressed in the literature. Researchers in some subfields, such as suicide prevention $^{2}$ and vaccination, have given sustained attention to the possibility of adverse 'iatrogenic' effects. However, in many areas of public health research, the picture is highly unclear: most systematic reviews do not extract data on adverse effects, and those that do often find little or no evidence. ${ }^{3}$ In this respect, public health contrasts markedly with clinical medicine, where there is a substantial literature on adverse events and patient safety, and the Hippocratic injunction to 'do no harm' is arguably more salient.

It is clearly important for people implementing and evaluating public health interventions to consider the possibility of unintended effects, particularly adverse effects. However, limited guidance is available on how to approach this question in a structured way. The aim of this paper is to illustrate five types of harms that may potentially be brought about by public health interventions in order to begin the process of formulating an analytical framework to understand harms. The paper focuses on public health interventions; clinical medicine and non-health policy sectors, which present rather different challenges, are not included here, although the latter would be a useful focus of further work. The categorisation presented here is not comprehensive, and not all adverse effects measured in evaluations may be readily assignable to a category; nonetheless, it may be of value as an initial broad framework for thinking about potential harms, and the wider impacts of public health policy interventions.

\section{DIRECT HARMS}

In some cases, desired health outcomes may have directly harmful effects, regardless of the content of the intervention targeting them. For example, sun exposure is associated with a reduced risk of some cancers. ${ }^{4}$ This implies that skin cancer prevention programmes which successfully reduce sun exposure may risk inadvertently increasing the risk of other cancers. Similarly, programmes to increase sports participation may increase injury risk. More generally, in many cases the evidence for the longer-term health impacts of behaviour change is lacking, as witnessed by the recent controversy over body weight and mortality sparked by the work of Flegal et al. ${ }^{5}$

\section{PSYCHOLOGICAL HARMS}

A more indirect category of harms is the possibility of negative psychological impacts on individuals as a result of interventions. Perhaps, most obviously, some population screening programmes may produce high numbers of false-positive results, potentially leading to substantial adverse effects in terms of psychological stress and unnecessary treatment; ${ }^{6-10}$ the NHS Health Check programme has recently been criticised on these grounds. ${ }^{11}$ Some universal psychological interventions, such as 'debriefing' after traumatic events, may have adverse mental health impacts. ${ }^{12}$ The dissemination of health messages through educational or media campaigns may generate damaging feelings of worry or guilt, which can have negative effects not only on general well-being but, in many cases, on the targeted behaviours themselves. ${ }^{13}{ }^{14}$ While most media campaigns do now try to avoid explicitly guilt-oriented messages, there is still considerable potential for harms, which have rarely been investigated systematically.

More indirect psychological harms may result where targeted health behaviours are bound up with individuals' social identity or relationships with others. It is hard to say how serious this potential for adverse effects may be, since such socio-psychological harms are rarely considered in evaluations of interventions. However, the qualitative literature suggests that, for example, moderate consumption of alcohol or other drugs may facilitate social interactions, ${ }^{15}{ }^{16}$ and that unprotected sex may facilitate trust and intimacy within sexual relationships. ${ }^{17}{ }^{18}$ It is thus possible that interventions targeting these behaviours could have negative impacts on social or intimate relationships. 


\section{EQUITY HARMS}

Interventions may create harm by worsening health inequalities. That is, some successful interventions may improve outcomes across the population, but exacerbate existing inequalities by benefiting privileged groups more than disadvantaged groups. ${ }^{19-}$ 21 This has particularly been a concern with individualistic or 'responsibilising' approaches to health promotion which, it is often argued, tend to benefit those who are in least need of them. ${ }^{22}$ It has also been argued that targeted or 'high-risk' approaches are more likely than population-level initiatives to widen health inequalities. ${ }^{24}$ Although the evidence base on such 'intervention-generated inequalities' is limited in extent, there is fairly reliable evidence that some interventions do widen inequalities, particularly media campaigns for smoking cessation. ${ }^{25}$

Equity harms raise complex ethical and methodological questions, since they may exist even where no individual in the population is worse off as a direct result of the intervention. However, given the evidence that inequality at a societal level is itself harmful across the population as a whole, ${ }^{26}$ it is clear that effects on equity are an important dimension of the potential harms of interventions.

\section{GROUP AND SOCIAL HARMS}

Group-based interventions may inadvertently create harms by singling out a particular subset of the population, or by the effects of bringing them together. A particular concern in the literature has been the so-called 'deviancy training' effect in group-based targeted interventions with young people for outcomes such as antisocial behaviour or drug use. That is, group interventions may generate harms by facilitating social interaction between people who are already partially socialised into marginal or 'deviant' norms, ${ }^{27} 28$ although the empirical evidence is mixed. ${ }^{29}$

Targeting particular groups or behaviours for intervention may contribute to stigmatising them. This may operate at an individual level, for example, in interventions targeting obesity, ${ }^{30-32}$ or on a larger scale, where the targeting of social interventions at disadvantaged groups or areas may exacerbate the stigma experienced by their members or residents. It may also contribute to divisions between groups, as in the case of alcohol restrictions in Aboriginal communities in Australia. ${ }^{33} 34$

More broadly, interventions may have negative impacts at the level of social norms or perceptions. For example, advocacy to promote bicycle helmets may contribute to an exaggerated perception of the injury risks of cycling, and hence lower cycling rates. ${ }^{35}$ This type of harm overlaps with those already discussed to the extent that interventions may contribute to culturally entrenched stereotypes of, for example, drug users or people from socio-economically deprived areas, hence contributing to the broader disadvantage which these groups may suffer. Social narratives-such as those which determine the boundaries of 'appropriate' behaviour by pregnant women (eg, around light consumption of alcohol)-may cause stress and guilt even where their putative health rationales receive limited support from the evidence. ${ }^{36}$ Such narratives may also lead people to reject public health messages as irrelevant to them, for example, by perpetuating stereotypes of particular health risks as associated with lower or marginal status. Finally, the broader impacts of 'medicalisation', for example, of mental health problems, on social norms, should also be borne in mind as a potential adverse effect of preventive programmes. ${ }^{37}$

\section{OPPORTUNITY COST HARMS}

A final category of potential harms relates to the opportunity costs of interventions, that is, the potential benefits which may be forgone as a result of committing resources to ineffective or less effective interventions, or to less serious public health problems. Such opportunity harms cannot be precisely defined, since the counterfactuals on which they rest are imponderable by their nature, and are not limited to specific prioritisation scenarios. Nonetheless, from some perspectives such opportunity harms considerably outweigh the other categories of harms discussed above, and they should be considered in any discussion of adverse effects. Indeed, many of the other harms mentioned above-particularly in the context of large, costly intervention programmes (eg, health checks and media campaigns) - may also represent substantial opportunity cost harms.

\section{CONCLUSIONS}

Whether intended or unintended, direct or indirect, interventions of any kind are likely to have wider effects than usually acknowledged by evaluators. For ethical and methodological reasons, it is imperative that the harmful effects of interventions are considered, collected and if possible alleviated by evaluators and designers of interventions. This paper has attempted to suggest categories which may be useful to diagnose and analyse types of unintended harms which may result from public health interventions.

The purpose of this paper is largely to stimulate debate and reflection, rather than to provide conclusive answers. It is not based on robust systematic review methods, and our use of the available data is selective. As already noted, many potential harms are not investigated in any depth in the empirical evaluation literature, and we have extrapolated from theory and qualitative data where necessary to fill these gaps; this should be borne in mind in interpreting the claims made in the paper. Some readers may consider some of our examples controversial or overstated, and it is true that most are probably not major systemic issues, with the possible exception of equity harms and, more debatably, opportunity harms. Nonetheless, there is good reason to think that all these types of harm may have substantial impacts in some cases.

Our framework suggests that many potential adverse effects may concern impacts which are diffuse and hard to measuresuch as attitudes, emotional reactions, or social relationships or norms-rather than the more tractable health status or behavioural outcomes which are usually the focus of public health evaluation research. While evaluations should continue to consider the possibility of adverse effects on the latter type of outcome, a broader scope may be required to achieve a fuller understanding of the total impact of interventions. With the possible exception of equity harms, ${ }^{38}$ a generalist 'box-ticking' approach to considering harms is unlikely to be of benefit. Rather, we would encourage researchers and practitioners to think as broadly as possible about the potential range of impacts before implementing or evaluating any intervention or policy. Our framework suggests that a wide range of preventive interventions may risk creating adverse harmful effects, and that their identification and measurement are a real gap in the literature. 


\section{What is already known on this subject}

- It is possible that public health interventions may sometimes create harms of various kinds.

- There is limited empirical data on the potential adverse effects of public health interventions, or theoretical discussion of what form such adverse effects might take.

\section{What this paper adds}

- The potential harms of public health interventions can be classed into the following categories: direct harms; psychological harms; equity harms; group and social harms; and opportunity harms.

- Taking adverse effects into account will often require the inclusion of broader and less tangible impacts than those which form the focus of conventional outcome evaluations.

Contributors Both the authors contributed to the conception and drafting of the paper, and approved the final version.

Competing interests None.

Provenance and peer review Not commissioned; externally peer reviewed.

\section{REFERENCES}

1 Macintyre S, Petticrew M. Good intentions and received wisdom are not enough. J Epidemiol Community Health 2000;54:802-3.

2 Gould MS, Marrocco FA, Kleinman M, et al. Evaluating iatrogenic risk of youth suicide screening programs. JAMA 2005;293:1635-43.

3 Ogilvie $D$, Foster $C E$, Rothnie $H$, et al. Interventions to promote walking: systematic review. BMJ 2007:334:1204.

4 Van der Rhee H, De Vries E, Coebergh J, et al. Does sunlight prevent cancer? A systematic review. Eur J Cancer 2006;42:2222-32.

5 Flegal KM, Kit BK, Orpana $\mathrm{H}$, et al. Association of all-cause mortality with overweight and obesity using standard body mass index categories: a systematic review and meta-analysis. JAMA 2013;309:71-82.

6 Bell R, Petticrew M, Luengo $S$, et al. Screening for ovarian cancer: a systematic review. Heal Technol Assess 1998;2.

7 Elmore JG, Barton MB, Moceri VM, et al. Ten-year risk of false positive screening mammograms and clinical breast examinations. N Engl J Med 1998;338:1089-96.

8 Lafata JE, Simpkins J, Lamerato L, et al. The economic impact of false-positive cancer screens. Cancer Epidemiol Biomarkers Prev 2004;13:2126-32.

9 Lerman C, Trock B, Rimer BK, et al. Psychological and behavioral implications of abnormal mammograms. Ann Intern Med 1991;114:657-61.

10 Lidbrink E, Elfving J, Frisell J, et al. Neglected aspects of false positive findings of mammography in breast cancer screening: analysis of false positive cases from the Stockholm trial. BMJ 1996;312:273-6.
11 Goodyear-Smith F. Government's plans for universal health checks for people aged 40-75. BMJ 2013;347:f4788.

12 Rose S, Bisson J, Churchill R, et al. Psychological debriefing for preventing post traumatic stress disorder (PTSD). Cochrane Database Syst Rev. Published Online First: 22 Apr 2002. doi:10.1002/14651858.CD000560

13 Ruiter RA, Abraham C, Kok G. Scary warnings and rational precautions: a review of the psychology of fear appeals. Psychol Heal 2001;16:613-30.

14 Cho H, Salmon CT. Unintended effects of health communication campaigns. J Commun 2007;57:293-317.

15 Järvinen M. Youth Drinking Cultures: European experiences. Aldershot: Ashgate, 2007.

16 Parker HJ. Illegal Leisure Revisited: Changing patterns of alcohol and drug use in adolescents and young adults. London: Routledge, 2011.

17 Rhodes T, Cusick L. Love and intimacy in relationship risk management: HIV positive people and their sexual partners. Sociol Health IIIn 2000;22:1-26.

18 Bolton M, McKay A, Schneider M. Relational influences on condom use discontinuation: a qualitative study of young adult women in dating relationships. Can J Hum Sex 2010;19:91-104.

19 Whitehead M, Dahlgren G. Concepts and Principles for Tackling Social Inequities in Health: Levelling Up Part 1. Copenhagen: WHO Regional Office for Europe, 2006.

20 Graham H, Kelly MP. Health Inequalities: Concepts, frameworks and policy. London: Health Development Agency, 2004.

21 Tugwell P, Savigny D de, Hawker G, et al. Applying clinical epidemiological methods to health equity: the equity effectiveness loop. BMJ 2006;332:358-61.

22 Barbones S, White M, Adams J, et al. How and why do interventions that increase health overall widen inequalities within populations? In: Barbones S, ed. Health, Inequality and Public Health. Bristol: Policy Press, 2009:65-83.

23 Macintyre S. Health inequalities in Scotland: What are they and what can we do about them? Glasgow: MRC Social and Public Health Sciences Unit, 2007.

24 Capewell S, Graham H. Will cardiovascular disease prevention widen health inequalities? PLOS Med 2010;7:e1000320.

25 Lorenc $T$, Petticrew M, Welch V, et al. What types of interventions generate inequalities? Evidence from systematic reviews. J Epidemiol Community Health 2013;67:190-3.

26 Wilkinson R, Pickett K. The Spirit Level: Why more equal societies almost always do better. London: Allen Lane, 2009.

27 Kaminer Y. Challenges and opportunities of group therapy for adolescent substance abuse: A critical review. Addict Behav 2005;30:1765-74.

28 Dishion TJ, McCord J, Poulin F. When interventions harm: Peer groups and problem behavior. Am Psychol 1999;54:755.

29 Weiss B, Caron A, Ball S, et al. latrogenic effects of group treatment for antisocial youth. J Consult Clin Psychol 2005;73:1036.

30 Puhl RM, Heuer CA. Obesity stigma: important considerations for public health. Am J Public Health 2010;100:1019-28.

31 MacLean L, Edwards N, Garrard M, et al. Obesity, stigma and public health planning. Heal Promot Int 2009:24:88-93.

32 Rukavina P, Li W. School physical activity interventions: do not forget about obesity bias. Obes Rev 2008;9:67-75.

33 D'Abbs P. Problematizing alcohol through the eyes of the other: Alcohol policy and aboriginal drinking in the Northern Territory, Australia. Contemp Drug Probl 2012;39:371.

34 Hudson S. Alcohol Restrictions in Indigenous Communities and Frontier Towns. St Leonards, NSW: Centre for Independent Studies, 2011.

35 Goldacre B, Spiegelhalter D. Bicycle helmets and the law. BMJ 2013;346:f3817.

36 Kelly YJ, Sacker A, Gray R, et al. Light drinking during pregnancy: still no increased risk for socioemotional difficulties or cognitive deficits at 5 years of age? J Epidemiol Community Health 2012;66:41-8.

37 Bramesfeld A, Platt L, Schwartz FW. Possibilities for intervention in adolescents' and young adults' depression from a public health perspective. Health Policy 2006;79:121-31.

38 Welch V, Petticrew M, Tugwell P, et al. PRISMA-Equity 2012 extension: reporting guidelines for systematic reviews with a focus on health equity. PLoS Med 2012;9: e1001333. 